\title{
Pre-Stressor Interference Control and Intrusive Memories
}

\author{
Johan Verwoerd · Ineke Wessel • Peter J. de Jong • \\ Maurice M. W. Nieuwenhuis • Rafaele J. C. Huntjens
}

Published online: 19 October 2010

(C) The Author(s) 2010. This article is published with open access at Springerlink.com

\begin{abstract}
Although intrusive imagery is a common response in the aftermath of a stressful or traumatic event, only a minority of trauma victims show persistent reexperiencing and related psychopathology. Individual differences in pre-trauma executive control possibly play a critical role. Therefore, this study investigated whether a relatively poor pre-stressor ability to resist proactive interference in working memory might increase risk for experiencing undesirable intrusive memories after being exposed to a stressful event. Non-clinical participants $(N=85)$ completed a modified version of a widely used test of interference control in working memory (CVLT; Kramer and Delis 1991) and subsequently watched an emotional film fragment. Following presentation of the fragment, intrusive memories were recorded in a 1-week diary and at a follow up session 7 days later. A relatively poor ability to resist proactive interference was related to a relatively high frequency of film-related intrusive memories. This relationship was independent of neuroticism and gender. These findings are consistent with the idea that a pre-morbid deficit in the ability to resist proactive interference reflects a vulnerability factor for experiencing intrusive memories after trauma exposure.
\end{abstract}

Keywords Intrusive memories - Proactive interference . Executive control · Trauma-film paradigm

J. Verwoerd $(\bowtie) \cdot$ I. Wessel · P. J. de Jong ·

M. M. W. Nieuwenhuis - R. J. C. Huntjens

Department of Clinical Psychology, University of Groningen,

Grote Kruisstraat 2-1, 9712 TS Groningen, The Netherlands

e-mail: j.r.l.verwoerd@rug.nl

\section{Introduction}

Although intrusive imagery is a common response in the aftermath of a stressful or traumatic event, only a minority of trauma victims show persistent re-experiencing and related psychopathology (Rothbaum et al. 1992). Considering this, one of the key issues in research on post-trauma information processing concerns individual differences in vulnerability. Inspired by recent findings in experimental memory literature, the present study focuses on the possible role of pre-trauma executive control in the frequency of intrusive remembering after experiencing a traumatic event. Executive control reflects a collection of separable meta-level abilities that regulate other (lower level) cognitive processes in the service of goal-directed behavior. Recent developments in the experimental memory literature (Friedman and Miyake 2004; Miyake et al. 2000) stress the importance of investigating separate (instead of general) executive abilities in applied research settings. Using general measures for examining the role of executive control in psychopathology may obscure the detection of specific underlying pathogenic mechanisms.

One particular executive ability can be described as resistance to proactive interference (PI). Resistance to PI refers to the ability to remove information that is no longer relevant from working memory (Friedman and Miyake 2004, for other executive abilities see Friedman et al. 2008; Miyake et al. 2000; Hedden and Yoon 2006). As an example of the ability to resist PI, let us consider the first weeks after purchasing a new mobile phone. During this period, it would be difficult to access the new number in long-term memory, because the extensively used old number will persistently intrude into conscious awareness. Flexible access to the memory representation of the new number would require a well-developed ability to resist 
unwanted interference from the old number. Interestingly, using latent-variable analysis, Friedman and Miyake (2004) found a relationship between resistance to PI and self-reported general intrusive thoughts. This raises the question of whether this specific executive ability of resistance to PI may also contribute to the gradual decrease of intrusive imagery that generally arise in the immediate aftermath of trauma (a prominent example of PI in real life). That is, a deficient ability to resist PI may complicate a rapid decline of these intrusive memories and may thus constitute a vulnerability factor for their persistence. In line with this hypothesis, a recent crosssectional study showed that a relatively good ability to resist PI was associated with fewer self-reported intrusive memories related to an earlier experienced stressful life event (Verwoerd et al. 2009). This earlier retrospective finding gives some support to the notion that a deficient ability to resist interference from irrelevant material in working memory may set people at risk for persistent intrusions in the aftermath of a stressful or traumatic event. A critical question that remains is whether these individual differences in executive functioning already exist prior to experiencing trauma.

Two lines of evidence support the notion that an inefficient ability to resist PI may act as pre-morbid risk factor. First, the results of a large twin study involving more than 500 adolescents showed that individual differences in general executive control, as well as in specific executive abilities, were almost entirely genetic in origin (Friedman et al. 2008). Second, using a stressful film fragment as an analogue stressor (cf. Holmes and Bourne 2008), a recent prospective study showed a relationship between pre-film resistance to $\mathrm{PI}$ (measured via $\mathrm{AB}-\mathrm{AC}$ list-learning) and intrusive thoughts measured $24 \mathrm{~h}$ after the presentation of the film (Wessel et al. 2008). These findings provide additional support for the proposed role of a reduced ability to resist PI in the persistence of intrusive memories after trauma. However, both earlier studies (i.e., Verwoerd et al. 2009; Wessel et al. 2008) used the same measure (i.e., paired-associates learning) to index resistance to PI. Perhaps the associations between performance on this measure and intrusive cognition were due to unforeseen task-specific factors rather than the higher-level executive ability of resistance to PI.

Therefore, the main goal of the present study was to replicate and extent the earlier finding of Wessel et al. (2008) using a modified version of the California Verbal Learning Test (CVLT) as an index of resistance to PI and a trauma film as analogue of real-life trauma (see Holmes and Bourne 2008 for a review of the use of trauma-films in analogue research). The CVLT is a widely used neuropsychological test to asses multiple aspects of verbal learning and memory (Kramer and Delis 1991; Delis et al.
1987). In earlier neuropsychological studies, several indices of the CVLT (including resistance to PI) have been successfully used to differentiate trauma-exposed people with PTSD from people without the disorder (e.g., Vasterling et al. 1998, 2002). The CVLT requires participants to study two lists of words (List 1 and List 2) consisting of different semantic categories (e.g., animals, vegetables). Some categories are similar between lists (shared categories) while others change from the first- to the second list (unshared categories). In line with earlier studies on the CVLT, the present study used the decrease in recall performance between the first and second list shared category words as an index of the ability to resist PI. A conceptual replication of the earlier results of Wessel et al. (2008) with decrease in recall performance as index of resistance to PI (instead of cued recall) would imply that it is unlikely that the relationship between resistance to PI and intrusive memories depends on taskspecific parameters.

Second, in addition to Wessel et al. (2008), the present study also explored the quality and modalities of filmrelated intrusions. Qualitative research has shown that the majority of trauma-related intrusive memories usually consist of the unwanted occurrence of sensory fragments or images of trauma that are mostly experienced in the visual modality, but may also be experienced as sounds, bodily sensations or feelings (see Ehlers et al. 2004; Holmes and Mathews 2010). To test whether indeed, the majority of the reported intrusions could be described as an image experienced in the visual modality (cf. Ehlers et al. 2004; Hackmann et al. 2004; Holmes and Mathews 2010), participants completed a tabular diary between the first and the second test session 7 days later. In this diary, they were instructed to record spontaneous intrusions (both images and verbal thoughts) of the film at the moment they entered into consciousness awareness. At the follow-up session 7 days after film viewing, participants delivered their diaries and completed a detailed questionnaire about the intrusiveness of the film memory during the past week.

A third and final goal of the current study was to investigate the role of neuroticism. Some theoretical models have suggested an interaction between attentional/ executive control and neuroticism in the prediction of anxiety (e.g., Derryberry and Reed 2002). That is, people with low levels of attentional/executive control, together with high levels of neuroticism are expected to show an increased risk of developing pathological anxiety. In line with this idea, the second goal of the present study was to investigate whether the influence of a weakened ability to resist interference from working memory on persistent intrusive remembering is especially pronounced in individuals with high levels of neuroticism. 


\section{Method}

Participants

Eighty-five undergraduate students participated in this study ( 20 men and 65 women $^{1}$; mean age 20.53 years, age range 18-50). All participants gave their written informed consent to take part. The University of Groningen Behavioral Research Ethical Committee granted ethics approval for this study. Participants were tested individually and were given course credits for participating.

\section{Stressful Film Fragment}

Participants were shown a 9-min fragment of the movie "Irreversible" produced by Gaspar Noé. The fragment shows a gruesome and brutal murder in a dark nightclub (see Nixon et al. 2007 for a trauma-film study using a different fragment from the same movie). The film fragment was projected on a large $(150 \times 120 \mathrm{~cm})$ screen in a darkened room.

\section{Resistance to PI}

As an index of the ability to resist PI from working memory, we used a modified version of an interference subtest of the California Verbal Learning Test (CVLT; Delis et al. 1987). In a recent psychometric study with healthy adults, the CVLT was found to possess good testretest validity (range $0.80-0.84$; Woods et al. 2006). Two word lists were constructed. List 1 contained 10 names of vegetables, 10 names of animals, and 10 names of a flowers category. List 2 contained 10 new names of vegetables, 10 new names of animals, and 10 names of musical instruments. Thus, the lists shared the categories animals and vegetables but they did not share the categories flowers and musical instruments. The order of the lists was counterbalanced between subjects. Words on both lists were matched on frequency and typicality of category membership using frequency and association norms from the Dutch language (Baayen et al. 1993; Ruts et al. 2004). ${ }^{2}$

\footnotetext{
1 An original sample of 115 participants took part in an extended version of the present experiment. Twenty participants were nonnative speakers of the Dutch language and therefore not used in the analyses reported in the present study. Another ten participants were excluded for the following reasons: five participants did not show up at the follow-up session; three participants were excluded because of equipment failure during the CVLT and another two participants complained about feeling unwell (i.e., headache, flu) at the time of testing.

${ }^{2}$ Part of the set of (Dutch) words selected for the current study was used in an earlier study carried out by one of the current authors (Huntjens et al. 2003).
}

In the present study, the procedure for learning the two word lists was as follows. First, the 30 words of the first list were presented on the computer screen for $1,000 \mathrm{~ms}$ each, with a $1,000 \mathrm{~ms}$ inter-stimulus interval. Participants were told that they should try to encode the words to the best of their ability, in order to recall them subsequently. The learning phase was followed by a 4-min free recall test (trial 1). During free recall, participants were asked to speak out loud their responses, which were tape recorded for later analyses. Next, encoding and free recall of firstlist words was repeated once more (trial 2). Participants were encouraged to encode and recall more words than during the first trial. After this, the words of the second list were presented in the same way as in trial 1 of the first list. Again, participants were given $4 \mathrm{~min}$ to recall these words.

As a measure of PI, CLVT scores were used for calculating an interference index (see Kramer and Delis 1991).This index accounts for the influence that the relative proportion of shared and non-shared category items recalled over two consecutive List 1 trials may have on the potential for PI during List 2 recall. Thus, the first step in calculating the interference index was computing a weighted average of correct recall of shared category members on trial 1 of List 1 (i.e., (total recall trial $1 \times($ recall shared items trial $1+$ trial2) $) /($ total recall trial $1+$ total recall trial 2). Thus, the total number of items recalled on trial 1 (i.e., both shared and unshared category members) was multiplied by the total number of shared category members that were recalled on trial 1 and 2 . The outcome of this multiplication was divided by the total number of items recalled on trial 1 and 2 (i.e., both shared and unshared category members). The second step in calculating the interference index was that the number of shared items recalled from list 2 was subtracted from the weighted average score. All in all, higher values of the interference index reflect stronger interference from List 1 shared items.

\section{Neuroticism}

Neuroticism was measured with the Five Factor Personality Inventory (FFPI; Hendriks et al. 1999) which is a selfreport questionnaire for measuring the five super ordinate factors of temperament. The emotional stability scale of the FFPI consists of 20 items (e.g., "Is afraid to do something wrong") answered on a five-point scale $(1=$ does not at all apply to me, $5=$ does very much apply to me). For half of the items, the direction was reversed, so that higher scores would indicate higher neuroticism. The internal consistency of the emotional stability scale used in this study was high (Cronbach's $\alpha=0.93$ ). 
Intrusive Memory Measures

Impact of Movie Scale (IMS). The wording of the Dutch version of the Impact of Event Scale (IES, Horowitz et al. 1979; Van der Ploeg et al. 2004) was adapted for the purpose of measuring film-related intrusions (for earlier studies using a similar adaptation of the IES see Holmes et al. 2009; Lang et al. 2009). Participants were instructed to rate the items with reference to the film fragment they had seen 7 days earlier. Four items from the original IES were dropped because they referred to symptoms unlikely to occur after watching a stressful film (e.g., "I felt as if it had not happened or it was not real", "My feelings about the event were kind of numb", "I tried not to talk about $i t$ "). Thus, the IMS consisted of 11 items (e.g., "Images came up spontaneously"). Items were rated on a four-point scale (not-at-all, rarely, sometimes, often) with assigned item weights of $0,1,3,5$ (cf. Horowitz et al. 1979), resulting in a total score ranging from 0 to 55). Internal consistency in the present sample was good (Cronbach's $\alpha=0.90)$.

Intrusion diary. Participants were asked to use a small diary $(15 \times 21 \mathrm{~cm})$ to record any film-related intrusions during 1 week following the film (e.g., Davies and Clark 1998; Holmes et al. 2004). Individual intrusions were registered on separate pages in the diary. Participants described the content of a specific intrusion (i.e., "what was the intrusion about?") and were asked to specify the unwanted memory as "image", "thought", "feeling/emotion" or "something else". Furthermore, participants also indicated the amount of distress, vividness, and level of control they experienced for each intrusive memory on $100 \mathrm{~mm}$ VAS scales. Elaborate verbal and written instructions were given about the nature of unwanted intrusions and how to keep the diary. Participants were asked to record every intrusive memory related to the film as soon as possible upon their occurrence. Participants were informed that people in the general population show large variation in the frequency of intrusive memories related to stressful events, which indicated that returning an empty diary with no film-related intrusions, would be as likely to occur as returning a diary with a large number of intrusions. The main dependent variables were the total number of intrusions reported in the diary and the modality in which the individual intrusions were experienced.

\section{Control Measures}

Film exposure and distress. In order to measure the amount of attention paid to the film fragment, participants rated the percentage of time they had looked away from the screen during the most intense part (the murder, 2 min) of the fragment on a $100 \mathrm{~mm}$ Visual Analog Scale (VAS;
$0=$ not at all $-100=$ all of the time). They also rated (VAS; $0=$ not at all $-100=$ extremely) film-related distress. Furthermore, participants were asked whether they had seen the stressful fragment before (Yes/No).

Prior trauma. The Negative Life Events and Trauma Questionnaire (NLETQ; Morgan and Janoff-Bulman 1994) was used as a screening instrument to check the frequency of earlier stressful/traumatic life events. These included verbal, physical, and sexual abuse, parental death, serious accidents, serious illness, and natural disasters. The variable of interest was the number of prior traumatic events with a self-reported intensity above 50 (range 0-100).

Diary compliance. Participants rated how well they had been able to complete the diary in line with the instructions on a $100 \mathrm{~mm}$ Visual Analogue Scale ("Please rate on the scale below to what extent you kept good record of your intrusions in the diary"; VAS, anchors $0=$ bad and $100=$ very good). To prevent conceivable demand effects on this measure, it was emphasized that participants' responses would not have any consequences for the assignment of course credits.

Depressive symptoms. In the current study, we used a Dutch translation of the Center for Epidemiologic Studies Depression Scale (CES-D; Bouma et al. 1995). The CES-D contains 20 items addressing depressive symptoms during the past week. Scores for each item range from 0 (rarely or none of the time) to 3 (most or all of the time). The maximum score on the CES-D is 60. Four items are worded positively and then scored reversed for the total CES-D score. The present study showed that the internal consistency of the CES-D was high (Cronbach's $\alpha=0.93$ ).

Learning ability. Possibly, increased use of learning strategies over time interferes with the CVLT's sensitivity to measure first-list interference. To control for the influence of a generally improved ability to retrieve information from memory over time (e.g., increased use of specific learning strategies), we calculated an index reflecting the percentage increase in recall performance between the number of correctly reported first- and second-list unshared words (see Table 1; cf. Kramer and Delis 1991).

\section{Procedure}

The present study consisted of two sessions separated by 1 week. Participants were recruited online via the website of a first-year course. For ethical reasons, participants were told in advance (on the website) that they would be presented with a film fragment with emotional content. Upon arrival at the test room, they filled in the FFPI and completed the CVLT. After this, the distressing fragment was presented. Participants were told that they were free to leave the room and to terminate the experiment at any point (all participants watched the complete fragment). 
Table 1 Mean, standard deviation and range of the main variables used in the present study

\begin{tabular}{lrrrcc}
\hline Variable & $N$ & Mean & SD & Min & Max \\
\hline CVLT shared List1-trial 1 & 85 & 10.8 & 2.6 & 5 & 17 \\
CVLT shared List 2 & 85 & 9.9 & 2.6 & 5 & 16 \\
CVLT unshared List1-trial 1 & 85 & 5.9 & 1.7 & 2 & 9 \\
CVLT unshared List 2 & 85 & 6.4 & 1.5 & 3 & 10 \\
CVLT interference & 85 & 1.1 & 2.2 & -4.50 & 6.04 \\
FFPI-neuroticism & 85 & 43.2 & 10.0 & 22 & 79 \\
Film-related distress (VAS) & 85 & 63.1 & 22.8 & 0 & 100 \\
Percent of time looked away & 85 & 18.5 & 31.7 & 0 & 100 \\
IMS-total & 85 & 4.6 & 5.0 & 0 & 21 \\
Diary intrusions & 85 & 1.6 & 1.8 & 0 & 7 \\
Diary compliance & 85 & 83.9 & 15.8 & 26 & 100 \\
CES-D-depression & 85 & 7.1 & 6.9 & 0 & 29 \\
Prior trauma & 85 & 1.3 & 1.5 & 0 & 6 \\
Learning ability & 85 & 18.6 & 1.7 & -50 & 167 \\
\hline
\end{tabular}

CVLT, California verbal learning test; FFPI-neuroticism, five factor personality inventory: neuroticism scale; IMS-total, impact of movie scale-total score; CES-D, center for epidemiologic studies depression scale; percent of time looked away, percent of time looked away during the most intense moments of the stressful film (2 min); prior trauma, number of earlier traumatic events with an impact above 50 (scale 0-100); learning ability, percentage increase in recall performance between the first trial of list 1 and list 2

Following the presentation of the fragment, they completed the film exposure and distress ratings. Following this, the experimenter gave instructions about the diary and the follow-up session 7 days later. At follow up, participants delivered their diaries, filled in the compliance ratings, completed the IMS and CES-D, and filled in the prior trauma screening list. ${ }^{3}$

\section{Data Reduction and Statistical Analyses}

The main analyses of the current study consisted of two Hierarchical Multiple Regression (HMR) analyses with the IMS and number of diary intrusions as dependent variables. Predictors were entered in three steps. The first step contained control variables. Selection of these variables was based on preliminary analyses described in the relevant paragraph in the results section. The second step of the analyses contained the CVLT interference score and the FFPI. The third step was included to investigate the interaction between resistance to PI and neuroticism. For that purpose the CLVT and FFPI scores were centered. The product of the centered scores was calculated and entered in the analyses (cf. Aiken and West 2000).

\footnotetext{
3 Participants also completed two measures of attention (Rapid Serial Visual Presentation [RSVP]) which are not reported here.
}

\section{Results}

\section{Descriptive Results}

Inspection of the distribution of the diary variable revealed a significant deviation from normality. Hence, a squareroot transformation was applied on the diary scores, resulting in a significantly improved distribution (Skewness and Kurtosis $\sim 1$ ). None of the other variables showed significant deviations from normality. Descriptive statistics for the variables used in the present study are shown in Table 1. In general, participants rated the film fragment as moderately stressful and were generally able to prevent looking away during the most threatening moments of the fragment. Furthermore, approximately $62 \%$ of the participants had experienced one or more prior traumatic or highly stressful events which is comparable with earlier epidemiological studies (56\%, Kessler et al. 1995; 67\%, Bernat et al. 1998). Finally, participants' subjective ratings indicated high compliance with the instructions to keep the 7-day diary.

Only three participants (3.5\%) had seen the film fragment previously. Analyses with and without these participants did not significantly change the main findings and conclusions. Therefore, we did not remove these persons from further analyses. Earlier studies (e.g., Kramer and Delis 1991) that investigated PI with the CVLT showed a between-lists decrease in performance for shared trials (normal interference) and a between- lists increase in performance for unshared trials. Overall, the current results were generally in line with these findings: Recall performance for shared items on list $2(M=9.9 ; \mathrm{SD}=2.6)$ decreased in comparison with shared items on the first trial of list $1(M=10.8 ; \mathrm{SD}=2.6), t(84)=3.5, P<0.01$. On the other hand, recall performance for the non-shared items showed the opposite pattern, with better recall performance on list $2(M=6.4 ; \mathrm{SD}=1.7)$ than on the first trial of list 1 $(M=5.9 ; \mathrm{SD}=1.5), t(84)=2.7, P<0.01$.

\section{Descriptive Analyses of Diary Intrusions}

Fifty-two participants (61\%) recorded at least one intrusive memory in their diaries, which was less than the 63 participants with intrusions on the IMS (75\%). Overall mean VAS ratings for stressfulness $(M=28.0, \mathrm{SD}=27.5)$, vividness $(M=48.2, \mathrm{SD}=27.8)$ and level of control $(M=57.7, \mathrm{SD}=32.4)$ revealed that participants were able to control the impact of their analogue intrusions related to the stressful film quite well. Inspection of the modalities of the recorded diary intrusions revealed that $80 \%$ of the reported intrusions could be described as mental image: $60 \%$ were experienced as a visual imagebased intrusion, $14 \%$ were experienced as a feeling or 
emotion and $6 \%$ as a combination of both (cf. Holmes and Mathews 2010). Twenty percent of the diary intrusions were experienced as a verbal thought. These results are generally in line with earlier clinical investigations of the phenomenology of intrusive remembering after real-life trauma (cf. Ehlers et al. 2004).

\section{Correlations Between Predictor Variables}

As shown in Table 2, point-biserial correlations with resistance to PI revealed that the strength of the interference effect on the CVLT was dependent of gender (male $>$ females) and the list order participants were assigned to (list order $21>$ list order 12). Additionally, the productmoment correlation between resistance to PI and the learning ability index was significant and film-related distress showed a negative association with the interference score. Hence, to take into account variation in intrusive re-experiencing unrelated to the ability to resist PI from working memory, we included gender, version, learning ability and film-related distress in the first step of the regression models. As depression and prior trauma did not show any association with either our interference index or the indices of intrusive memories (see Table 2), we decided not to include these variables in the regression analyses. Overall, the correlations presented in Table 2 show that multicollinearity was no reason for concern.

\section{Relationship Between Resistance to PI (CVLT) and Intrusive Memories (IMS-total and Diary Intrusions) ${ }^{4}$}

As shown in Table 3, the first step of the HMR analyses with the IMS-scores as the dependent variable resulted in a significant $13 \%$ of explained variance for the four control variables, $F(4,80)=2.8, P<0.05$. Inclusion of resistance to PI and neuroticism in the second step resulted in an additional $10 \%$ of explained variance in intrusive memories. This result was almost completely based on the significant effect of resistance to PI, semi-partial $r=0.33$, $P<0.05$. A relatively weak ability to resist interference in working memory resulted in a relatively high frequency of film-related intrusive memories in the week after the

\footnotetext{
$\overline{4}$ In contrast with real-life traumatic events which usually leave behind an intrusive trauma memory in the whole group of trauma victims, the impact of the trauma film in the present analogue design was not strong enough to elicit intrusive memories in every participant (21 participants reported zero intrusions). To better model reality, we repeated the main analyses in the subsample of 64 participants who reported at least one instance of intrusive reexperiencing. Results did not differ from the whole group analyses, yet the unique effects for resistance to PI in the prediction of IMS and diary intrusions were clearly more pronounced (IMS: $\beta=0.45$, semipartial $r=0.38$. $P<0.01$; Diary: $\beta=0.36$, semi-partial $r=0.30$, $P<0.05)$.
}

presentation of the stressful film. Neuroticism did not explain any unique variance in IMS scores. Finally, the inclusion of the interaction term between resistance to PI and neuroticism in the third step of the model did not result in any extra explained variance in the IMS scores.

The HMR analysis with the number of diary intrusions as the (transformed) dependent variable resulted in a nonsignificant $6 \%$ of explained variance for the control variables in the first step (see Table 3). The second step resulted in a significant contribution in explained variance for the interference score, semi-partial $r=0.24, P<0.05$ with a marginal significant effect for neuroticism, semipartial $r=0.21, P=0.08$. The interaction term in the third step of the model did not result in any extra explained variance in the number of diary intrusions.

\section{Discussion}

The present analogue study examined whether a poor executive ability existing prior to a stressful or traumatic event would increase the probability of experiencing intrusive memories afterwards. The main results can be summarized as follows. First, the specific executive ability of resistance to PI (as indexed by the CVLT) predicted the frequency of intrusive re-experiencing in the week following exposure to a trauma film. Second, prospective effects of the ability to resist PI from entering working memory were not associated with prior trauma and depressive symptoms and were independent of learning ability, gender and version effects on the CVLT task. Third, the relationship between resistance to PI and intrusive memories did not depend on high trait levels of neuroticism. Hence, no evidence was found for the suggestion that people with a combination of low resistance to PI and high levels of neuroticism would posses an increased risk of persistent intrusions after trauma (cf. Derryberry and Reed 2002). Fourth, the current study investigated the qualities of intrusive re-experiencing in a 1week diary. The results showed that the majority of the recorded diary intrusions were experienced as visual images of the earlier trauma film. This finding concurs with earlier qualitative research involving trauma-related intrusive memories that mainly consisted of brief sensory-mostly visual-fragments of the traumatic experience (Ehlers et al. 2004; Hackmann et al. 2004; Ehlers and Steil 1995; Holmes and Mathews 2010).

The present findings replicate and extend earlier prospective results of Wessel et al. (2008). Using a different paradigm (CVLT) and an independent sample, our conceptual replication provides more consistent evidence for the role of a specific executive ability in the prediction of intrusive cognition. This specific executive function was the ability to resist PI in working memory. A more specific 
Table 2 Pearson product-moment and point-biserial correlations between the predictor variables $(N=85)$

\begin{tabular}{|c|c|c|c|c|c|c|c|c|c|}
\hline & List order & Gender & $\begin{array}{l}\text { CVLT } \\
\text { interference }\end{array}$ & Neuroticism & $\begin{array}{l}\text { Film-related } \\
\text { distress }\end{array}$ & Depression & Prior trauma & $\begin{array}{l}\text { Learning } \\
\text { ability }\end{array}$ & IMS \\
\hline Gender & - & 1 & & & & & & & \\
\hline CVLT interference & $0.34 * *$ & $0.28 * *$ & 1 & & & & & & \\
\hline Neuroticism & 0.17 & $0.26^{*}$ & 0.10 & 1 & & & & & \\
\hline Film-related distress & 0.14 & 0.14 & $-0.24 *$ & 0.16 & 1 & & & & \\
\hline Depression & $0.24 *$ & 0.01 & 0.13 & $0.64 * *$ & 0.14 & 1 & & & \\
\hline Prior trauma & 0.11 & 0.09 & -0.08 & $0.27 *$ & 0.08 & $0.24 *$ & 1 & & \\
\hline Learning ability & $0.36 * *$ & 0.02 & $-0.33 * *$ & -0.02 & $0.23 *$ & -0.03 & -0.04 & 1 & \\
\hline IMS & $-0.26^{*}$ & 0.20 & 0.13 & 0.18 & 0.19 & 0.14 & 0.18 & 0.18 & 1 \\
\hline Diary Intrusions & -0.10 & 0.15 & 0.12 & 0.19 & 0.21 & 0.02 & 0.06 & -0.06 & $0.70 * *$ \\
\hline
\end{tabular}

CVLT California verbal learning test, IMS impact of movie scale

$\dagger P<0.10 ; * P<0.05 ; * * P<0.01$

focus on underlying pathogenic mechanisms is important, because a failure to specify precise underlying mechanisms may lead to null- or inconsistent findings in studies investigating the role of executive control in psychopathology. In the context of intrusive cognitions, this might have been the case with studies using working memory capacity (WMC) which is a rather general index of executive control. WMC is defined as the extent to which goalrelevant information is kept in an active state in working memory, despite the interference of irrelevant information (Unsworth and Engle 2007). There are some analogue studies that have investigated the relationship between WMC and stressor-related intrusive cognition. One retrospective study found the predicted negative relationship between WMC and intrusive memories of a stressful personal event (Klein and Boals 2002). However, two recent prospective studies, using trauma films as analogue stressors, failed to find the expected association (Nixon et al. 2007; Wessel et al. 2008, experiment 1). Considering this, the fact that we found a significant relationship between resistance to PI and intrusive cognition in two earlier independent samples (Verwoerd et al. 2009; Wessel et al. 2008), suggests that the selection of indices of specific (instead of general) executive abilities might lead to more consistent results and conclusions in studies investigating the role of executive control in psychopathology.

Our findings regarding the role of resistance to PI are generally consistent with previous neuropsychological research comparing PTSD patients and trauma-exposed controls on a wide range of cognitive measures. Several studies, focusing specifically on indices of proactive and retroactive interference, found recall deficits in PTSD patients when initial learning was taken into account (Uddo et al. 1993; Vasterling et al. 1998, 2002; Yehuda et al. 1995; see also Vasterling and Brailey 2005). Moreover, Vasterling et al. (1998) found that the tendency to make intrusion errors on cognitive interference tasks was positively correlated with re-experiencing symptoms. These clinical results may be very informative about underlying pathogenic mechanisms such as resistance to PI. However, it remains unclear whether neuropsychological abnormalities are a consequence of PTSD or represent a pre- morbid risk (cf. Vasterling and Brailey 2005). Considering this, the current prospective findings support the possibility that these cognitive abnormalities may already exist prior to trauma and may thus function as a pre-morbid risk factor for the development and maintenance of PTSD.

There are several issues that deserve further attention. First, the present study used a variant of the trauma-film paradigm to provide a laboratory model for individual differences in intrusive re-experiencing (see Holmes and Bourne 2008 for a review). Studies using analogue stressors and populations are open to criticism that the studied processes are dissimilar to those observed in the aftermath of an actual trauma. In the laboratory, ethical restrictions do not allow presenting participants with extremely stressful material. Thus, these analogue studies provide the opportunity of investigating the influence of risk factors on intrusions in a prospective design, but at the same time potentially limit external validity. Some support for the validity of the current findings can be found in the quality of the recorded diary intrusions, which were generally in line with earlier clinical investigations of intrusive memories after trauma (Ehlers et al. 2004; Hackmann et al. 2004). However, these laboratory findings surely have to be extended to other methodologies with survivors of actual traumatic events. More specifically, future work should directly compare analogue intrusions elicited by a traumafilm and autobiographical intrusions related to real-life stressors in the same sample.

Second, observation of the number of IMS- and diary intrusions showed that $75 \%$ of the participants had scores 
Table 3 Summary of hierarchical regression analyses of IMS scores and diary intrusions $(N=85)$

\begin{tabular}{|c|c|c|c|c|c|c|c|}
\hline Step & Predictors & $(\Delta) R^{2}(\%)$ & $d f$ & $F$-(change) & $B$ & SE $B$ & Beta \\
\hline \multicolumn{8}{|c|}{ Model 1: IMS } \\
\hline \multirow[t]{4}{*}{1} & Gender & 13 & 4,80 & $2.77 *$ & 1.64 & 1.39 & 0.13 \\
\hline & Film-related distress & & & & 0.02 & 0.03 & 0.08 \\
\hline & CVLT-list-order & & & & -1.24 & 1.27 & -0.12 \\
\hline & Learning ability & & & & 0.54 & 0.33 & 0.20 \\
\hline \multirow[t]{6}{*}{2} & Gender & 10 & 2,78 & $4.62 *$ & 2.04 & 1.41 & 0.18 \\
\hline & Film-related distress & & & & 0.03 & 0.03 & 0.12 \\
\hline & CVLT-list-order & & & & -2.05 & 1.28 & -0.22 \\
\hline & Learning ability & & & & 0.02 & 0.01 & 0.20 \\
\hline & Neuroticism & & & & 0.02 & 0.06 & 0.05 \\
\hline & CVLT-interference & & & & 0.79 & 0.28 & $0.34 * *$ \\
\hline \multirow[t]{7}{*}{3} & Gender & 0.3 & 1,57 & 0.24 & 2.23 & 1.52 & 0.18 \\
\hline & Film-related distress & & & & 0.03 & 0.03 & 0.12 \\
\hline & CVLT-list-order & & & & -2.27 & 1.31 & -0.22 \\
\hline & Learning ability & & & & 0.79 & 0.30 & 0.20 \\
\hline & Neuroticism & & & & 0.03 & 0.06 & 0.05 \\
\hline & CVLT-interference & & & & 0.79 & 0.30 & $0.34 * *$ \\
\hline & Neuroticism*CVLT-interference & & & & 0.04 & 0.77 & 0.01 \\
\hline \multicolumn{8}{|c|}{ Model 2: Diary intrusions-sqrt } \\
\hline \multirow[t]{4}{*}{1} & Gender & 6 & 4,80 & 0.92 & 0.40 & 0.52 & 0.09 \\
\hline & Film-related distress & & & & 0.02 & 0.01 & 0.19 \\
\hline & CVLT-list-order & & & & -0.39 & 0.50 & 0.11 \\
\hline & Learning ability & & & & -0.01 & 0.01 & -0.12 \\
\hline \multirow[t]{6}{*}{2} & Gender & 8 & 2,78 & $3.80 *$ & 0.22 & 0.53 & 0.05 \\
\hline & Film-related distress & & & & 0.02 & 0.01 & 0.21 \\
\hline & CVLT-list-order & & & & -0.71 & 0.50 & -0.19 \\
\hline & Learning ability & & & & -0.01 & 0.01 & -0.10 \\
\hline & Neuroticism & & & & 0.05 & 0.03 & $0.27 \dagger$ \\
\hline & CVLT-interference & & & & 0.21 & 0.11 & $0.26^{*}$ \\
\hline \multirow[t]{7}{*}{3} & Gender & 1 & 1,77 & 0.67 & 0.26 & 0.54 & 0.06 \\
\hline & Film-related distress & & & & 0.02 & 0.01 & 0.20 \\
\hline & CVLT-list-order & & & & -0.74 & 0.51 & -0.20 \\
\hline & Learning ability & & & & -0.01 & 0.01 & -0.11 \\
\hline & Neuroticism & & & & 0.06 & 0.03 & 0.29 \\
\hline & CVLT-interference & & & & 0.19 & 0.11 & 0.23 \\
\hline & Neuroticism*CVLT-interference & & & & -0.22 & 0.29 & -0.10 \\
\hline
\end{tabular}

IMS impact of movie scale, CVLT California verbal learning test

$\dagger P<0.10 ; * P<0.05 ; * * P<0.01$

above zero on the IMS while only $61 \%$ of the participants reported at least a single instance of intrusive re-experiencing in the diary. One explanation for this unexpected finding would be that the items of the IMS which also include the symptom of avoiding the trauma memory (e.g., "I tried not to think of it."). However, closer inspection of the items specifically referring to intrusive re-experiencing showed a similar pattern with more participants scoring above zero on the IMS. An alternative explanation for discrepancies in intrusion reports on the IMS and diary measures may be that filling in a diary constitutes a suboptimal method for registering intrusions. Even though ratings of compliance with the diary instructions were high, the retrospective nature of those compliance ratings precludes firm conclusions as to the validity of the diary reports. For example, we have no way of knowing whether participants failed to report intrusions or whether they really had their intrusions on the specific day corresponding to the diary page they used to report that intrusion. Especially the latter is important given the idea that the 
ability to resist PI would help the gradual decrease of intrusion occurrence over time. Future studies might use more sophisticated measures (e.g., electronic devices, online registration) for keeping track of the time course of the intrusions. In addition, advanced analytical methods may then be used for testing the moderating effects of resistance to $\mathrm{PI}$ on individual intrusion trajectories (see Roberts et al. 2006, for an example involving the time course of overgeneral memories).

Third, the current study found no moderating effect of high levels of neuroticism on the prospective relationship between resistance to PI and intrusive memories. Furthermore, the marginally significant main effect of neuroticism on the diary measure (no effect on the IMS) only partly supports earlier clinical work, showing that high levels of neuroticism increase the risk of developing PTSD (e.g., Lauterbach and Vrana 2001). Possibly, the sensitivity of the present analogue design may not have been sufficient for detection of the hypothesized interaction. However, it may also be the case that high neuroticism mainly influence intrusion-related qualities such as distress and experienced control instead of frequency. Yet, post-hoc correlation analyses between pre-film neuroticism and stressfulness ratings/experienced control of the diary intrusions did not support this hypothesis. Of course it is also possible that the non-significant interaction between resistance to PI and neuroticism was the result of limited statistical power for detecting an interaction with 85 participants. Future analogue and clinical work may further elucidate the precise characteristics of this proposed relationship.

Fourth, interestingly, some earlier studies made similar use of the stressful film paradigm to assess measures of vulnerability to PTSD symptoms. For instance, Holmes and colleagues showed that both the personality trait schizotypy as well as a data-driven processing style were associated with the number of intrusive memories from a trauma film (Holmes and Bourne 2008; Holmes et al. 2004; Steel and Holmes 2007). Future research may further examine whether these characteristics covary with low levels of resistance to PI.

In conclusion, the present results indicate that a relatively weak executive ability to resist interference from working memory may be involved in intrusive memories of stressful events. Moreover, our data suggest that this weakened ability already existed prior to the presentation of the stressor. These results support the idea that a premorbid deficit in the ability to resist PI from working memory may constitute a vulnerability factor for their persistence and subsequent development of PTSD. Future prospective work should test whether the current relationship would generalize to real traumatic events, for example by selecting groups with an increased risk for trauma exposure (e.g., soldiers before deployment to a war zone).
Acknowledgments We are grateful to Sophie Snoek for her help in data collection. This research was supported by an Innovational Research Incentive VIDI grant (452-03-329) of the Foundation for Behavioural and Educational Sciences of the Netherlands Organization for Scientific research (NWO) awarded to Ineke Wessel. Rafaele Huntjens was supported by an Innovational Research Incentive VENI grant (451-05-018) of the Foundation for Behavioural and Educational Sciences of the Netherlands Organization for Scientific research $(\mathrm{NWO})$.

Open Access This article is distributed under the terms of the Creative Commons Attribution Noncommercial License which permits any noncommercial use, distribution, and reproduction in any medium, provided the original author(s) and source are credited.

\section{References}

Aiken, L. S., \& West, S. G. (2000). Multiple regression. In A. E. Kazdin (Ed.), Encyclopedia of Psychology (pp. 350-352). Washington, DC: APA.

Baayen, R. H., Piepenbrock, R., \& van Rijn, H. (1993). The CELEX lexical data base on CDROM. Philadelphia: Linguistic Data Consortium.

Bernat, J. A., Ronfeldt, H. M., Calhoun, K. S., \& Arias, I. (1998). Prevalence of traumatic events and peri-traumatic predictors of posttraumatic stress symptoms in a nonclinical sample of college students. Journal of Traumatic Stress, 11, 645-664.

Bouma, J., Ranchor, A. V., Sanderman, R., \& Van Sonderen, E. (1995). Het meten van symptomen van depressie met de CES-D: Een handleiding [The measurement of symptoms with the CESD: A manual]. Rijksuniversiteit Groningen: Noordelijk Centrum voor Gezondheidsvraagstukken.

Davies, M., \& Clark, D. M. (1998). Predictors of analogue posttraumatic intrusive cognitions. Behavioral and Cognitive Psychotherapy, 26, 303-314.

Delis, D. C., Kramer, J. H., Kaplan, E., \& Ober, B. A. (1987). The California verbal learning test. San Antonio, TX: Psychological Corporation.

Derryberry, D., \& Reed, M. A. (2002). Anxiety-related attentional biases and their regulation by attentional control. Journal of Abnormal Psychology, 111, 225-236.

Ehlers, A., Hackmann, A., \& Michael, T. (2004). Intrusive reexperiencing in post-traumatic stress disorder: Phenomenology, theory, and therapy. Memory, 12, 403-415.

Ehlers, A., \& Steil, R. (1995). Maintenance of intrusive memories in posttraumatic stress disorder: A cognitive approach. Behavioural and Cognitive Psychotherapy, 23, 217-249.

Friedman, N. P., \& Miyake, A. (2004). The relations among inhibition and interference control functions: A latent-variable analysis. Journal of Experimental Psychology: General, 133, 101-135.

Friedman, N. P., Miyake, A., Young, S. E., DeFries, J. C., Corley, R. P., \& Hewitt, J. K. (2008). Individual differences in executive functions are almost entirely genetic in origin. Journal of Experimental Psychology: General, 2, 201-225.

Hackmann, A., Ehlers, A., Speckens, A., \& Clark, D. A. (2004). Characteristics and content of intrusive memories in PTSD and their changes with treatment. Journal of Traumatic Stress, 17, 231-240.

Hedden, T., \& Yoon, C. (2006). Individual differences in executive processing predict susceptibility to interference in verbal working memory. Neuropsychology, 20, 511-528.

Hendriks, A. A. J., Hofstee, W. K. B., De Raad, B., \& Angleiter, A. (1999). The five-factor personality inventory (FFPI). Personality and Individual Differences, 27, 307-325. 
Holmes, E. A., \& Bourne, C. (2008). Inducing and modulating intrusive emotional memories: A review of the trauma film paradigm. Acta Psychologica, 127, 553-566.

Holmes, E. A., Brewin, C. R., \& Hennessey, R. G. (2004). Trauma films, information processing, and intrusive memory development. Journal of Experimental Psychology: General, 133, 3-22.

Holmes, E. A., James, E. 1., Coode-Bate, T., \& Deeprose, C. (2009). Can playing the computer game "Tetris" reduce the build-up of flashbacks for trauma? A proposal from cognitive science. PLoS ONE, 4, e4153. doi:10.1371/journal.pone.0004153.

Holmes, E. A., \& Mathews, A. (2010). Mental imagery in emotion and emotional disorders. Clinical Psychology Review, 30, $349-362$.

Horowitz, M., Wilner, N., \& Alvarez, W. (1979). Impact of event scale: A measure of subjective stress. Psychosomatic Medicine, 41, 209-218.

Huntjens, R. J. C., Postma, A., Peters, M. L., Woertman, L., \& van der Hart, O. (2003). Interidentity amnesia for neutral episodic information in dissociative identity disorder. Journal of Abnormal Psychology, 112, 290-297.

Kessler, R. C., Sonnega, A., Bromet, E., \& Hughes, M. (1995). Posttraumatic stress disorder in the National Comorbidity Survey. Archives of General Psychiatry, 52, 1048-1060.

Klein, K., \& Boals, A. (2002). The relationship of life event stress and working memory capacity. Applied Cognitive Psychology, 15, $565-579$.

Kramer, J. H., \& Delis, D. C. (1991). Interference effect on the California Verbal Learning Test: A construct validation study. Psychological Assessment, 3, 299-302.

Lang, T. J., Mould, M. L., \& Holmes, E. A. (2009). Reducing depressive intrusions via a computerized cognitive bias modification of appraisals task: Developing a cognitive vaccine. Behaviour Research and Therapy, 47, 139-145.

Lauterbach, D., \& Vrana, S. (2001). The relationship among personality variables, exposure to traumatic events, and severity of posttraumatic stress symptoms. Journal of Traumatic Stress, $14,29-45$

Miyake, A., Friedman, N. P., Emerson, M. J., Witzki, A. H., \& Howerter, A. (2000). The unity and diversity of executive functions and their contributions to complex 'frontal lobe' tasks: A latent variable analysis. Cognitive Psychology, 41, 49-100.

Morgan, H. J., \& Janoff-Bulman, R. (1994). Positive and Negative self-complexity: Patterns of adjustment following traumatic versus non-traumatic life experiences. Journal of Social and Clinical Psychology, 13, 63-85.

Nixon, R. D. V., Nehmy, T., \& Seymour, M. (2007). The effect of cognitive load and hyper arousal on negative intrusive memories. Behaviour Research and Therapy, 45, 2652-2663.

Roberts, J. E., Carlos, E. L., \& Kashdan, K. B. (2006). Impact of depressive symptoms, self-esteem, and neuroticism on trajectories of overgeneral autobiographical memory over repeated trials. Cognition and Emotion, 20, 383-401.

Rothbaum, O. R., Foa, E. B., Riggs, D. S., Murdock, T., \& Walsh, W. (1992). A prospective examination of posttraumatic stress disorder in rape victims. Journal of Traumatic Stress, 3, 455-475.

Ruts, W., De Deyne, S., Ameel, E., Vanpaemel, W., Verbeemen, T., \& Storms, G. (2004). Dutch norm data for 13 semantic categories and 338 exemplars. Behavior Research Methods, Instruments \& Computers, 36, 506-515.

Steel, C., \& Holmes, E. A. (2007). The role of involuntary memories in posttraumatic disorder and psychosis. In J. Mace (Ed.), Involuntary memory (pp. 68-86). Oxford: Blackwell.

Uddo, M., Vasterling, J. J., Brailey, K., \& Sutker, P. B. (1993). Memory and attention in combat-related post-traumatic stress disorder (PTSD). Journal of Psychopathology and Behavioral Assessment, 15, 43-52.

Unsworth, N., \& Engle, R. W. (2007). The nature of individual differences in working memory capacity: Active maintenance in primary memory and controlled search from secondary memory. Psychological Review, 114, 104-132.

Van der Ploeg, E., Mooren, T. M., Kleber, R. J., Van der Velden, P. G., $\&$ Brom, D. (2004). Construct validation of the Dutch version of the impact of event scale. Psychological Assessment, 16, 16-26.

Vasterling, J. J., \& Brailey, K. (2005). Neuropsychological findings in adults with PTSD. In J. J. Vasterling \& C. R. Brewin (Eds.), Neuropsychology of PTSD: Biological, cognitive and clinical perspectives (pp. 178-207). New York, NY: The Guilford Press.

Vasterling, J. J., Brailey, K., Constants, J. I., \& Sutker, P. B. (1998). Attention and memory dysfunction in posttraumatic stress disorder. Neuropsychology, 12, 125-133.

Vasterling, J. J., Duke, L. M., Brailey, K., Constants, J. I., Allain, A. N., Jr., \& Sutker, P. B. (2002). Attention, learning, and memory performances and intellectual resources in Vietnam veterans: PTSD and no disorder comparisons. Neuropsychology, 16, 5-14.

Verwoerd, J. R. L., Wessel, I., \& de Jong, P. J. (2009). Individual differences in experiencing intrusive memories: The role of the ability to resist proactive interference. Journal of Behavior Therapy and Experimental Psychiatry, 40, 189-201.

Wessel, I., Overwijk, S., Verwoerd, J., \& de Vrieze, N. (2008). Prestressor cognitive control is related to intrusive cognition of a stressful film. Behaviour Research and Therapy, 46, 496-513.

Woods, S. P., Delis, D. C., Scott, J. C., Kramer, J. H., \& Holdnack, J. A. (2006). The California verbal learning test-second edition: Test retest reliability, practice effects, and reliable change indices for the standard and alternate forms. Archives of Clinical Neuropsychology, 21, 413-420.

Yehuda, R., Keefe, R. S. E., Harvey, P. D., Levengood, R. A., Gerber, D. K., Geni, J., et al. (1995). Learning and memory in combat veterans with posttraumatic stress disorder. American Journal of Psychiatry, 152, 137-139. 